\title{
Outcome strength of weightlifters, bilateral limb deficit, relative strength and its relationship to Women Arab Championships results (senior) 2017
}

\author{
Ahmed Awad ${ }^{1}$ \\ ${ }^{1}$ Department of Sports Training, Faculty of Physical Education, Damietta University, Egypt.
}

\begin{abstract}
This research aims to determine the women Arab championship relative strength for each lift. In addition, the perfect outcome strength percentage between the two limbs as a bilateral limb deficit indicators through the relationship between their results during the Arab championships 2017 and some physical tests. The descriptive approach was used to suit the nature and objectives of the research, the sample was selected intentional manner of the International Senior Arab Championship 2017, held in the period from 18-23 / 12/2017 in the Arab Republic of Egypt, which was represented in the first three places in each weight category championship 24 women average age (19.24 \pm 3.75). The researchers reached to find the Women Arab championship relationship between body weight, snatch, clean \& jerk and total lift. Also the relative strength of snatch, clean \& jerk, and total. Are being founded and the bilateral limb deficit between the forces generated from the two limbs are founded by equations and percentage and we recommend using this rate in developing training programs for juniors and selecting the squads in weightlifting.
\end{abstract}

Keywords. Concentric/overload eccentric, maximum strength, snatch lift.

\section{Introduction}

$\mathrm{I}$ mproving the maximum strength (MS) is considered one of the most important training 1 tasks of the training programs in all sports in general but in the training programs of weightlifting sport in particular. And it is the most important factor affecting the level of explosive power (Dietmar, 2010), which is in turn the most important factor affecting the success of performing the
Olympic lifts in weightlifting sport especially for the snatch lift (Ikeda et al., 2012; Kristof \& Chad, 2015; Pat, 2014; Schilling, 2002). Since it is a single maximal performance, in which the weightlifter raises. The bar from the ground to the position of stretched arms over the head in one continuous motion without any interruption (Benjamin et al., 2014; Jones \& John, 2010; Kalichova, 2016; Zachary, 2010).

Olympic weightlifting can be defined as a form of competition lifting that involves a contest of maximum strength levels in both lifts snatch and clean \& jerk (Thomas \& Roger, 2006), so that the muscle strength is very important and a critical factor in weightlifting that encourage us to make this study to identify muscular strength rate for Arab championships.

Muscular strength is the ability to generate maximum external force, recall that in mechanics and physics and force mean an instantaneous measure of two bodies' interaction and the force appear by one of following ways, first the movement of the body is changed, second it's a vector quantity. It is characterized be (a) magnitude, (b) direction, and (c) point of application (Zatsiosky \& Kraemer, 2006; Ebada, 2012). Also, muscular strength can define as the capacity of a muscle to expert maximally one time (Thomas \& Roger, 2006), it's also mean the muscle's ability to force external high resistance (Sobhy, 2000; Ahmed, 2017).

Relative strength (RS) is a comparative measure of strength based on variables like total body 
weight. (Thomas \& Roger, 2006), it can calculate for each lift by dividing the final number of each lift on the real weightlifter's weight body.

The bilateral limb deficit (BLD) defined as the difference between the maximal force of contracted muscles and it happens when one lateral muscle have a higher force than the other (Kuruganti \& Murphy, 2011).

Some say the BLD occurs when the force generated by both limbs together is different than the sum of the forces generated by the two each limb alone under the same experimental conditions (Kuruganti \& Murphy, 2008; Kuruganti \& Seaman, 2006; Ohtsuki, 1983).

In addition, we can describe the BLD as the difference in force generated by muscles when they have contracted alone, or together with the contralateral muscle group. A deficit happens when the summed unilateral force is higher than the bilateral force, and it's found in both males and females and in athletic and non-athletic individuals (Archontides \& Fazey, 1993; Jakobi \& Chilibeck, 2001; Ohtsuki, 1994; Henry \& Smith, 1961; Howard \& Enoka, 1991).

So that we can say that, the BLD happens in weightlifting when one of the two limbs and usually be the lower limb generates force higher than the upper limb causes failed in jerk the weight. It appears when we compare the sum weight lifted of (front squat and front press) against the front jerk push. Where it is in front, squat the weightlifter Produce maximum force possible from the legs. In addition, in the front press, the shoulders and arms Produce maximum force possible, but when the legs and the shoulders and arms work together in front jerk push they cannot produce the same quantity of force. It happens also when we compare the sum of (back squat and back press) against the back jerk push; it is not all will be equals although. The same muscles groups involved together in the performance of jerk push.

We hope in this research to find the perfect bilateral limb deficit rates between front legs muscles against front shoulders and arm muscles. As well finding the perfect bilateral $\operatorname{limb}$ deficit rates between the back legs muscles against back, shoulders, and arms. Also the Arabian rates and percentage between the third record of the snatch and the clean and jerk and the total.

\section{Methods}

The descriptive approach was used to suit the nature and objectives of the research. The sample was selected intentional manner through score sheet results of the International senior Arab Championship 2017. Which was held in the period 18-23/ 12/2017 in the Arab Republic of Egypt, which was represented in the first three places in each weight category championship for 24 women?

We got the results of women Arab championships (senior) 2017 senior from the final sheets of the competitions where I was working as a technical official, and the other measurements we measure it directly at the camp before the completion, we measure the tests for the first five weightlifters that expect to be the first three places between them.

By analysis, the records of first places weightlifters in each category $(48,53,58,63,69,75$, 90 and $90+)$ we got the number of finally successful attempts of the snatch, clean \& jerk and total for them, then calculating the statics for the results.

The correlation and linear regression between bodyweight and snatch, bodyweight and clean \& jerk, bodyweight and the total for both players in each category were found (all statics did with SPSS), also for bodyweight and the relative strength of the snatch, the clean and jerk, and the total.

Through the sum of front squat and front press, we found the BLD rate for the front jerk push, which represented the Arabian level also in the same way through the sum of back squat and back press we found the BLD rate for the back jerk push, which represented the Arabian level.

Through the BLD definition, the jerk push result must be the sum of the two lateral muscles group of lower and upper limbs. It means that the Outcome strength (OS) of front group muscles of legs appear in front squat as well the OS of front muscles group of shoulders and arm appear in the front press. Then if the two groups of muscles work together in front jerk push it must produce the sum of the two tests but it doesn't.

The configuration differences in bones and muscles between the legs and the arms imposes to be a difference in OS when they work together and that appears clearly in tests results so that the research aims two find the equation that describes 
the perfect relation between the both OS of two limbs through line regression.

Through the definition of RS, we calculated the RS for each lift by dividing the best number achieved for every lift on the real weightlifter's bodyweight.

We calculated the front BLD through measured the best number of front squat, front press, and front jerk push. Then we collect the two numbers of front squat plus front press next we get the percentage of front jerk push of this total lift. In addition, the same for black BLD, second step we compared the front BLD average with the back BLD average, which also must be equals to achieve balance during the performance.

\section{Statistical Analyses}

Statistical analysis was carried out using the SPSS and Excel programs were used to calculate the statistical formulas: standard deviation, average, linear regression, percentage and relative strength.

\section{Results}

The following table shows that it appears the correlation and Linear regression analyses between the bodyweights, the snatch, the clean and the total for women 48-90 categories.

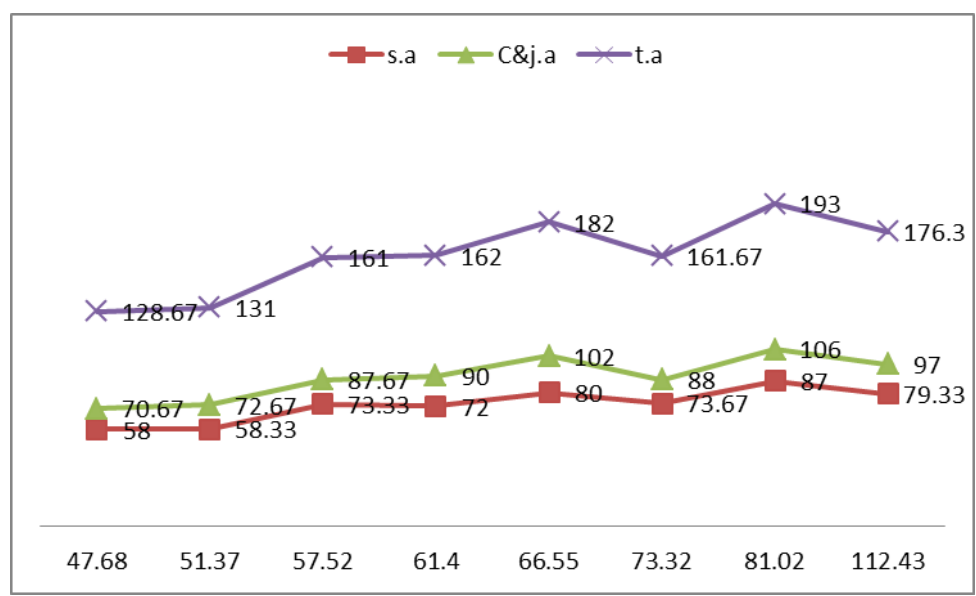

Figure 1. The relationship between bodyweight, the snatch, the clean and jerk and the total for women categories.

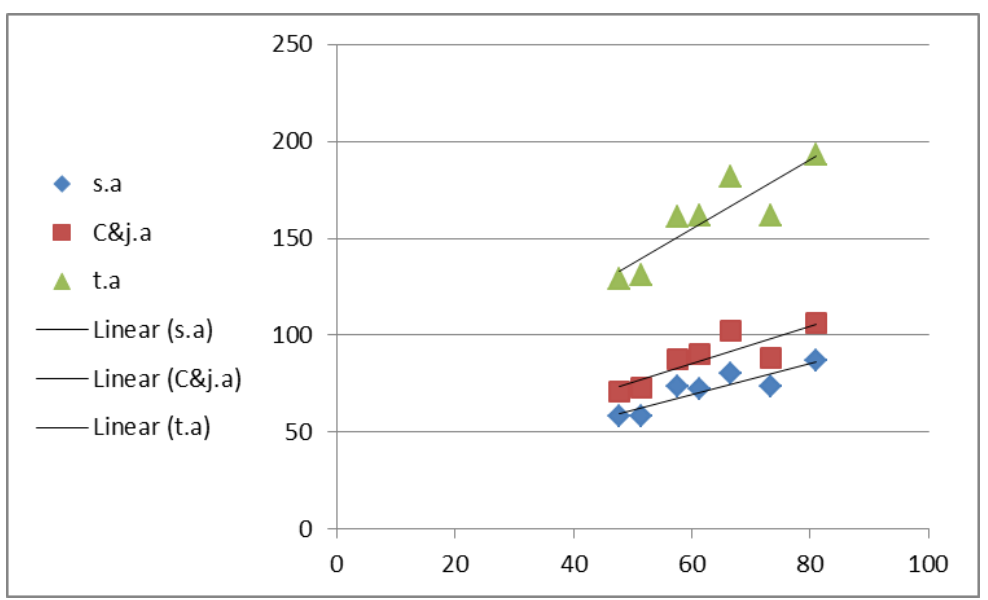

Figure 2. Linear regression analyses between the bodyweights, the snatch, the clean and the total for women 48-90 categories. 


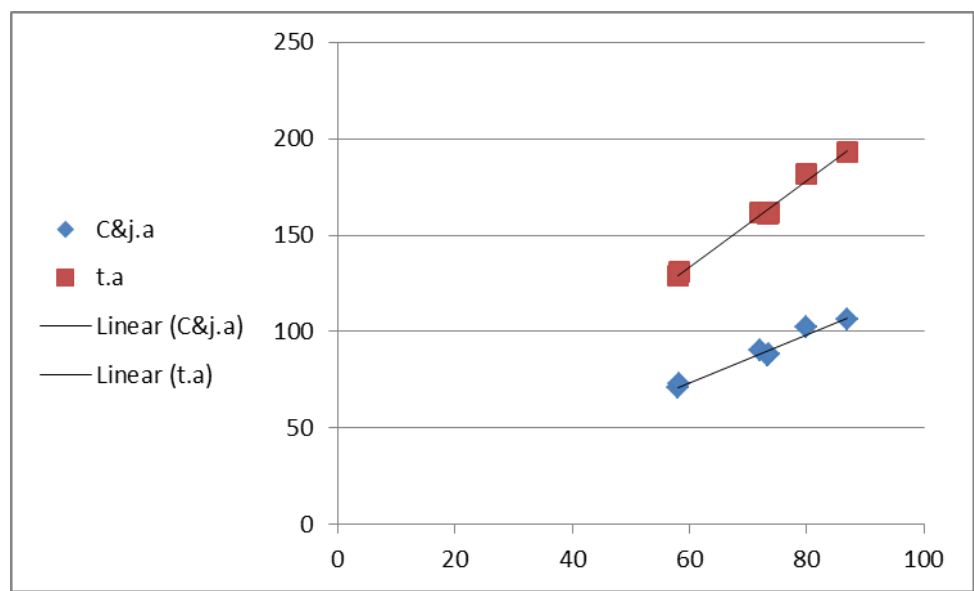

Figure 3. Linear regression analyses between the snatch, the clean and jerk and the total for women 48-90 categories.

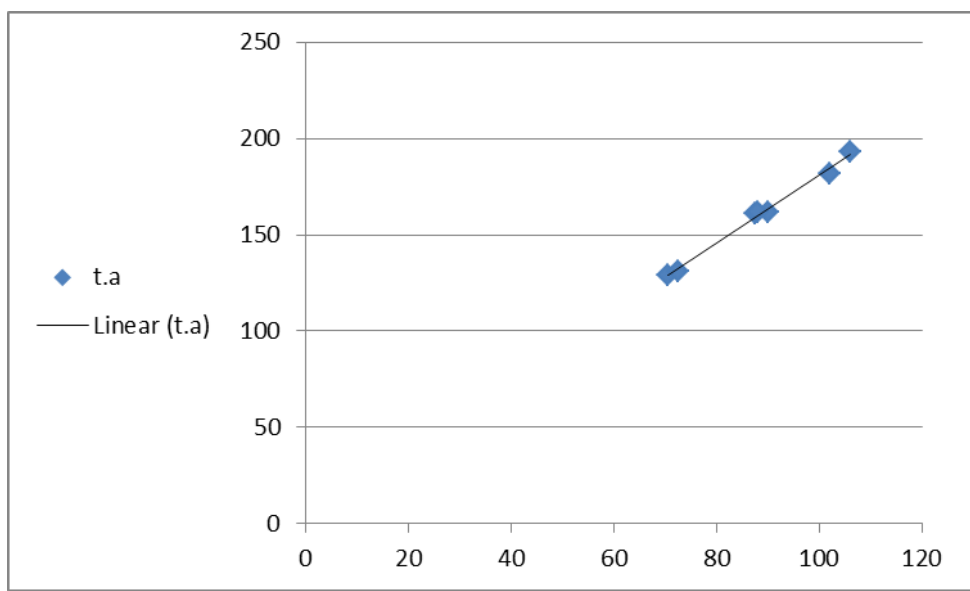

Figure 4. Linear regression analyses between the total and the clean and jerk for women 48-90 categories.

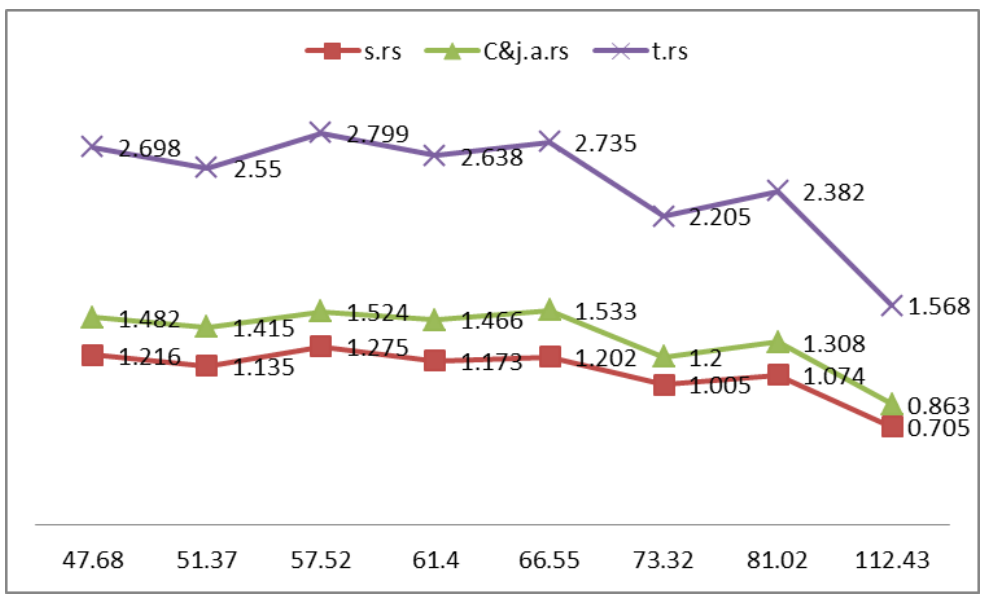

Figure 5. The relationship the between bodyweight, the snatch relative strength, the clean and jerk relative strength and the total relative strength for women categories. 


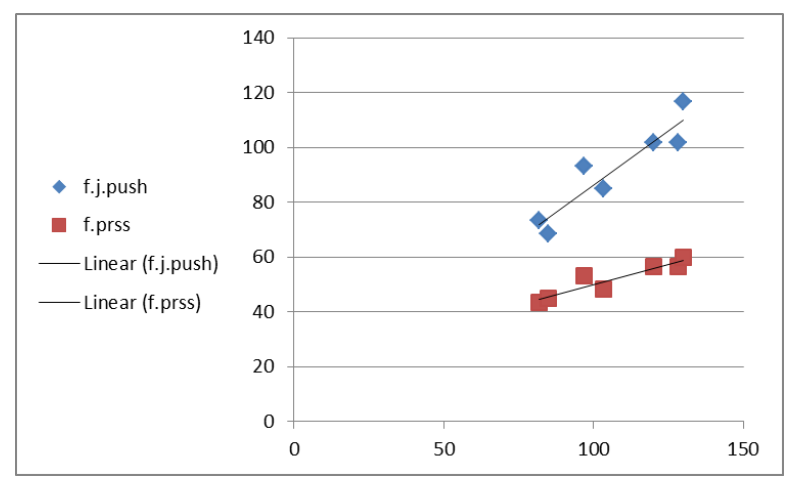

Figure 6. Linear regression analyses between front squat, front jerk push and front press for women 48-90 categories.

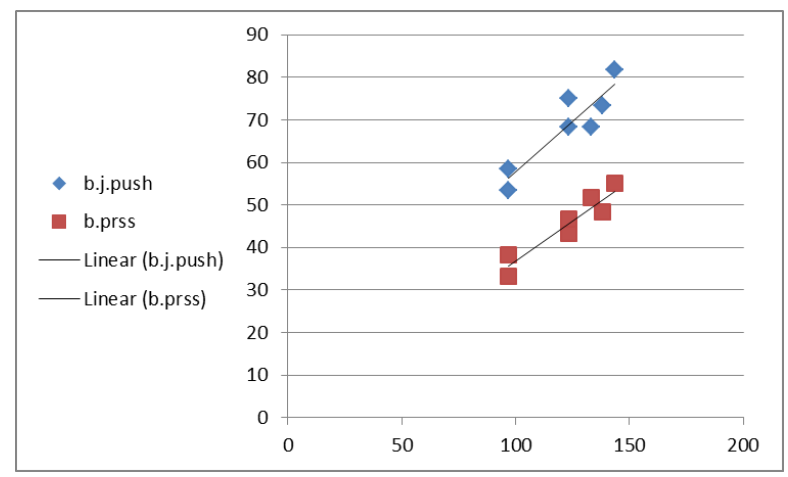

Figure 7. Linear regression analyses between back squat, back jerk push and back press for women 48-90 categories.

\section{Table 1}

Bilateral limb deficit for front jerk push against (front squat + front press) and bilateral limb deficit for back jerk push against (back squat + back press).

\begin{tabular}{ccccccc}
\hline Body weight & $\begin{array}{c}\text { Front squat }+ \\
\text { Front press }\end{array}$ & Front jerk & Front BLD Rate & $\begin{array}{c}\text { Back squat }+ \\
\text { back press }\end{array}$ & Back jerk & Back BLD Rate \\
\hline $47.68 \pm 0.20$ & $125 \pm 24.83$ & $63.33 \pm 12.47$ & $50.66 \%$ & $130 \pm 28.58$ & $53.33 \pm 12.47$ & $41.02 \%$ \\
$51.37 \pm 1.22$ & $130 \pm 20.08$ & $65 \pm 7.07$ & $50.00 \%$ & $135 \pm 22.80$ & $58.33 \pm 6.24$ & $43.21 \%$ \\
$57.52 \pm 0.18$ & $151.66 \pm 30.27$ & $76.67 \pm 4.71$ & $50.55 \%$ & $166.67 \pm 36.43$ & $68.33 \pm 6.24$ & $41.00 \%$ \\
$61.40 \pm 1.42$ & $150 \pm 21.72$ & $83.33 \pm 12.47$ & $55.55 \%$ & $170 \pm 42.33$ & $75 \pm 10.80$ & $44.12 \%$ \\
$66.55 \pm 1.54$ & $185 \pm 56.72$ & $86.66 \pm 19.43$ & $46.85 \%$ & $186.67 \pm 55.43$ & $73.33 \pm 8.49$ & $39.29 \%$ \\
$73.32 \pm 2.10$ & $176.66 \pm 36.99$ & $78.33 \pm 15.46$ & $44.34 \%$ & $185 \pm 38.71$ & $68.33 \pm 11.78$ & $36.94 \%$ \\
$81.02 \pm 2.81$ & $190 \pm 43.32$ & $95 \pm 17.80$ & $50.00 \%$ & $198.33 \pm 38.49$ & $81.66 \pm 6.24$ & $41.18 \%$ \\
\hline
\end{tabular}

It is clear from Table 1 that the statistical significance of the Bilateral limb deficit for front jerk push against (front squat + front press) and bilateral limb deficit for back jerk push against (back squat + back press).

\section{Discussion}

Figure 1 appears the correlation in the numbers of the snatch, the clean and jerk and the total of the following women categories 48, 53, 58, 63, 69, 75, 90 . Which were higher correlated that their relationship can be represented in a straight line so that we grouped it together, it appears to snatch $71.762 \pm$ 
9.827, the clean and jerk $88.143 \pm 12.303$ and the total $159.905 \pm 22.050$.

Figure 2 shows the linear regression between the bodyweight and the snatches for women Arab competition level, which can predict by this equation $\mathrm{y}=0.8059 \mathrm{x}+21.234$ (snatch $=0.8059$ * bodyweight +21.234 ). It appears also the relationship between the bodyweight and the clean and jerk by this equation $y=0.9658 x+27.597$ (clean $\&$ jerk $=0.9658 *$ bodyweight +27.597 ). And finally the relationship between bodyweight and total through the following equation $y=1.7717 x+48.831$ $\left(\right.$ total $=1.7717^{*}$ bodyweight +48.831$)$.

Figure 3 appears the relationship between snatch and clean \& jerk for Arab competition level $\mathrm{y}=$ $1.2337 x-0.3843$ (clean \& jerk $=1.2337 *$ snatch 0.3843 ), it shows also the relationship between the snatch and the total for the Arab competition level y $=2.2337 x-0.3843\left(\right.$ total $=2.2337{ }^{*}$ snatch -0.3843$)$.

Figure 4 the relationship between clean \& jerk and the total for Arab competition level $\mathrm{y}=1.7873 \mathrm{x}$ $+2.3658($ total $=1.7873 *$ clean $\&$ jerk +2.3658$)$.

Figure 5 appear the correlation between bodyweight and snatch RS, which was $1.098 \pm 0.168$, also the correlation between bodyweight and clean \& jerk RS $1.349 \pm 0.2122$ and the correlation between bodyweight total RS $2.447 \pm 0.392$ for women categories

We can note that whenever the weight increased and the bodyweight is constant the RS also increased so that the total RS is higher than snatch RS and the clean and jerk RS, it means that the relationship between them is positive and this agrees with the results of (Khaled, 2011; Khaled et al., 2015).

Figure 6 represents the relationship between front squat and front jerk push can be show from the next equation $\mathrm{y}=0.4961 \mathrm{x}+25.53$ (front jerk push $=0.4961 *$ front squat +25.53 ), and the relationship between front squat and front press can be show from the next equation $y=0.2993 x+20.05$ (front press $=0.2993 *$ front squat +20.05 ).

Figure 7 appears the relationship between back squat and back jerk push can be show from the next equation $\mathrm{y}=0.4719 \mathrm{x}+10.691$ (back jerk push $=$ $0.4719 *$ back squat +10.691 ), and the relationship between back squat and back press can be show from the next equation $\mathrm{y}=0.378 \mathrm{x}-0.928$ (back press $=0.378 *$ back squat -0.928 ).
The destination of two figures is to put the perfect rates of outcome strength between the two limbs which, if the coaching success to make the weightlifters reach it they will success in the six attempts they have at the completion.

The relationship between the front squat, the front press and the front jerk push is positive it means that if the front squat and the front press increased. It will lead to increase the front jerk; we can say that happens because the lateral muscles group in the arms and legs participates in the front jerk push, and the same for back squat and back press to the back jerk and this agrees with the results of (Andre, Nazareth, 2012)

Table 1 appears the bilateral limb deficit between the force generated of the two limbs and the perfect percentage of Arab championship women level for example, in 48 categories. If we collect the numbers of front squat plus front press, it will be (125.00). The front jerk push is (63.33), estimated at $(50.66 \%)$ and the front average for all categories estimated at $(49.85 \%)$, as well if we collect the numbers of back squat plus back press. it will be (130.00) and the front jerk push is (33.33) estimated at $(41.02 \%)$. The back average for all categories estimated at $(40.52 \%)$, we can notes that the deficit rates almost equal and it means that the whole weightlifters were the best at their categories and its indications of possibility to generalization this results for weightlifters.

This table represents the normal percentage that has already been the outcomes strength between the two limbs to clear the limits of bilateral limb deficit in weightlifting for Arab Women Weightlifters 2017.

The explanation of these rates demands on the definition of BLD as the difference between the maximal force of contracted muscles and it happens when one lateral muscle have a higher force than the other does. (Kuruganti \& Murphy, 2011). Therefore, the literal outcomes strength from the front legs and arms muscles through the physical tests, front squat and front press must be equals with the outcome strength from the front jerk push. Because the two muscles groups of arms and legs work together in the front jerk. That is one, and the front BLD percentage must be equal with the back BLD percentage that is the second, but when we look at the table, we see that the average front BLD $(49.85 \%)$ and when we compare it with the average back BLD $(40.52 \%)$. we find a huge deficit and this gap must be correct. 
This gap between outcomes strength of the two limbs may come from the interesting of weightlifters in training. The front muscles more than the backs. In addition, it can happen because the wrong beliefs that the weightlifters must train every day the front side of the body because it seems. That it bears the biggest burden on performance and finally we can say. That this gap is a result of bad training periodization so that we recommend repairing these errors.

\section{Conclusions}

The researchers reached to find the Arab championship relationship between the body weight, the snatch, the clean and jerk and the total, also the relative strength of the snatch, the clean and jerk and the total are being founded and the bilateral limb deficit between the forces generated from the two limbs are founded by equations and percentage

The researcher recommends that those rates should be taken into account when developing training programs for juniors and selecting the squads in Weightlifting

\section{References}

Ahmed A. Preparing coaches to made weightlifters, faculty of physical education. Damietta University, 2017.

Andre C, Nazareth Kh. Relevance of hand dominance to the bilateral deficit phenomenon. Eur J Appl Physiol, 2012; 112: 4163-4172.

Archontides C, Fazey JA. Inter-limb interactions and constraints in the expression of maximum force: a review, some implications and suggested underlying mechanisms. J Sports Science, 1993; 11: 145-158.

Ebada K, Abdel Hady I, El-Rouby M. Graphic Network to diagnose results of Clean and Jerk lifting a function some of Dynamic variables, relative strength and BMI for female Olympic weightlifters. International Journal of Humanities and Management Sciences, 2015; 3(2): 117122.
Ebada Kh. Weightlifting for juniors, faculty of physical education. Port Said University, 2012.

Ebada, Kh. Relative strength, body mass and height as predictors of Olympic weightlifting players' performance. Selcuk University Journal of Physical Education and Sports, 2011; 13(2): 166-171.

Henry FM, Smith LE. Simultaneous vs separate bilateral muscular contractions in relation to neural overflow theory and neuromotor specificity. Res Q Exerc Sport, 1961; 32: 42-46.

Howard JD, Enoka RM. Maximum bilateral contractions are modified by neurally mediated interlimb effects. J Appl Physiol, 1991; 70: 306-316.

Jacobi J, Chilibeck P. Bilateral and unilateral contractions: possible differences in maximal voluntary force. Can J Appl Physiol, 2001; 26: 12-33.

Kuruganti U, Murphy T. BLD expressions and myoelectric signal activity during submaximal and maximal isometric knee extensions in young, athletic males. Eur J Appl Physiol, 2008; 102: 721-726.

Kuruganti U, Seaman K. The bilateral leg strength deficit is present in old, young and adolescent females during isokinetic knee extension and flexion. Eur J Appl Physiol, 2006; 97: 322-326.

Ohtsuki, T. Changes in strength, speed and reaction time induced by simultaneous bilateral muscular activity. In: Swinnen SP, Heurer H, Massion J, Casaer P (Eds) Interlimb coordination: neural dynamic and cognitive constraints. San Diego: Academic Press, 1994; 259-274.

Ohtsuki T. Decrease in human voluntary isometric arm strength induced by simultaneous bilateral exertion. Behav Brain Res, 1983; 7: 165-178.

Sobhy H. Measurement and evaluation in physical education: Part 2. Dar Elfekr, 2000.

Thomas RB, Roger WE. Weight training steps to success, United States: Human Kinetics, 2006.

Usha K, Tiernan M. BLD phenomenon and the role of antagonist muscle activity during maximal isometric knee extensions in young athletic men. Eur J Appl Physiol, 2011; 111: 1533-1539.

Vladimir MZ, William JK. Science and practice of strength training. 2nd edition, United States: Human Kinetics, 2006. 